\title{
Variación en la actividad eléctrica de la corteza cerebral prefrontal, frontal y temporal en consumidores de Cannabis
}

\author{
Variation in the electrical activity of the prefrontal, frontal and temporal cortex \\ in Cannabis users
}

\author{
Ivone A. Castelblanco-Montañez ${ }^{1,2}$, Katherine Peña-Maldonado ${ }^{1,3}$ Carmen H. Moreno-Durán ${ }^{1,4}$
}

\begin{abstract}
Resumen
El presente estudio evaluó la variación en la actividad eléctrica de la corteza cerebral prefrontal, frontal y temporal en registros electroencefalográficos de estudiantes universitarios consumidores y residentes en la ciudad de Bogotá, durante y en ausencia de consumo de Cannabis, contrastado con una población control de no consumidores. Se evidenció que los efectos de Cannabis en la actividad eléctrica cerebral durante el consumo, ocasionan la presencia de una mayor actividad alfa, indicando estados de relajación y poca concentración; a su vez, un incremento en la actividad beta, vinculada a la captación de diversos estímulos y la disminución de la actividad delta tras la recompensa generada con el consumo. En ausencia de consumo, se manifiesta el aumento de las ondas delta con respecto a la población control, ondas asociadas con estados de ansiedad. Adicionalmente, se evidenció que el uso prolongado y frecuente de esta sustancia genera una tolerancia, al no variar la actividad cerebral durante y en ausencia del consumo de Cannabis, del mismo modo, la presencia en mayor proporción de ondas theta en ausencia de consumo frente a no consumidores, sugiere que tras el uso prologado de la sustancia los consumidores tienden a manifestar estados de somnolencia y adormecimiento constantes.
\end{abstract}

Palabras clave: Actividad eléctrica cerebral, corteza frontal y temporal, Cannabis, consumidores universitarios, tolerancia, abuso y dependencia

\begin{abstract}
This research assessed the variation in the electrical activity of the prefrontal, frontal and temporal on the cerebral cortex in EEG records from college Cannabis-user residents in Bogota, during and in the absence of Cannabis use, contrasted with a control population of non-users. It was evident that the effects of Cannabis in brain electrical activity during the consume cause the increase of alpha activity, indicating states of relax and low concentration; also, an increase in beta activity, related to the reception of different stimulus and decrease of delta activity generated by the rewarding after the use. In the absence of consumption, delta waves increasing in comparison with the control group, waves associated with anxiety states. Furthermore, it was noticed that the prolonged and frequent use of this substance generates tolerance, without variation in brain activity during and in the absence of Cannabis use. In the same way, the presence in a greater proportion of theta waves in the absence of consumption compared to nonusers, suggesting that after the prolonged use of the substance consumers tend to manifest constant sleepiness and drowsiness.
\end{abstract}

Key words: Brain electrical activity, Bogotá, Cannabis, tolerance, university consumers

\section{INTRODUCCIÓN}

Desde los años 70 se han desarrollado estudios empleando técnicas de radio-imagen, para identificar el deterioro de las funciones cognitivas asociado al consumo de Cannabis. Entre las técnicas empleadas está la electroencefalografía, una exploración funcional del sistema nervioso central (SNC) mediante la cual se obtiene el registro de la actividad eléctrica cerebral en tiempo real. La Federación Internacional de Sociedades

Recibido: agosto 2015; aceptado: abril 2017.

${ }^{1}$ Grupo de Investigación en Neurociencias (GINUD), Facultad de Ciencias y Educación, Licenciatura en Biología, Universidad Distrital Francisco José de Caldas. Bogotá, Colombia. ${ }^{3}<$ pmkaterine@gmail.com> ${ }^{4}<$ helenamd2000@gmail.com>.

Correo electrónico para correspondencia: ${ }^{2}<$ ivonecastel@gmail.com> 
de Electroencefalografía y Neurofisiología Clínica han recomendado un posicionamiento convencional de electrodos, llamado 10-20, conformado por 21 electrodos activos y 2 de referencia conectados en los lóbulos de las orejas (Sanei y Chambers 2007).

La señal del EEG mide las corrientes que fluyen durante excitaciones sinápticas de las dendritas de muchas neuronas piramidales de la corteza cerebral. Cuando se activan las células del cerebro (neuronas), las corrientes sinápticas que se producen dentro de las dendritas, generan un campo eléctrico secundario sobre el cuero cabelludo medible por los sistemas de EEG. Las diferencias de potenciales eléctricos son causadas por la suma de los potenciales postsinápticos graduales de células piramidales que crean dipolos eléctricos entre el soma (cuerpo de una neurona) y las dendritas apicales, que se ramifican a partir de las neuronas (Sanei y Chambers 2007).

Por lo tanto, sólo las grandes poblaciones de neuronas activas pueden generar suficiente potencial para ser registradas mediante los electrodos ubicados sobre el cuero cabelludo (Sanei y Chambers 2007), cuando se sincroniza la actividad eléctrica de un grupo importante de neuronas, se provoca una descarga que representa la suma de la fuerza de cada una de las neuronas integrantes de cada grupo. Esta sincronización ha dado lugar a que se reconozcan algunos patrones que, por su identidad en frecuencia y forma han dado origen a los distintos ritmos (Franco 2007). En un ser humano adulto y sano, las amplitudes y frecuencias de tales señales cambian de un estado a otro, tal como la vigilia y el sueño. Las características de las ondas también cambian con la edad (Franco 2007).

Existen cuatro ondas cerebrales que se distinguen por diferentes rangos de frecuencia. Estas ondas de bajas a altas frecuencias, se denominan, delta $(\delta)$, theta $(\theta)$, alfa $(\alpha)$, beta $(\beta)$. Las ondas delta se encuentran dentro de $0,5-$ $4 \mathrm{~Hz}$, se asocian principalmente con el sueño profundo y pueden estar presentes en el estado de vigilia. Las ondas theta se encuentran de 4 a $8 \mathrm{~Hz}$ (Constant y Sabourdin 2012), aparecen como descenso de conciencia hacia la somnolencia, se han asociado con el acceso a estados de inconciencia, inspiración creativa y la meditación profunda (Sanei y Chambers 2007). La frecuencia para las ondas alfa va de $8-13 \mathrm{~Hz}$, se asocian a una conciencia relajada y sin ninguna atención o concentración. Una onda beta está dentro del rango de 14 a $26 \mathrm{~Hz}$, es el ritmo habitual del estado de vigilia del cerebro, asociadas con el pensamiento y la atención (Sanei y Chambers 2007).
Fundamentado en lo anterior, la presente investigación estudió la variación en la actividad eléctrica de la corteza cerebral prefrontal, frontal y temporal en registros electroencefalográficos de estudiantes universitarios consumidores, durante y en ausencia de consumo de Cannabis, contrastado con una población control de no consumidores.

\section{MATERIALES Y MÉTODOS}

El registro de la actividad eléctrica de la corteza cerebral se realizó a 19 individuos, 16 de ellos estudiantes universitarios consumidores de Cannabis y 3 estudiantes universitarios controles no consumidores, la reducción de los participantes controles se debió a los costos de la técnica de registro. Las características de la población se presentan en la tabla 1. Entre los criterios de inclusión de la muestra poblacional, se tuvo en cuenta que fueran estudiantes universitarios, que estuvieran en un rango de edad entre 18 y 30 años, que no presentaran enfermedades clínicamente significativas o activas. Para el caso de los consumidores que fueran consumidores habituales de Cannabis y que no consumiera más de tres psicoactivos habitualmente. La población control se constituyó con personas que no consumieran ningún tipo de sustancias psicoactivas y que los estudiantes manifestaran interés y disposición para cumplir con los requisitos del estudio. A los estudiantes seleccionados se les entregó un consentimiento informado según el decreto 2164 de 1992 y la Ley 10 de 1990 en la Resolución 008430 de 1993 en el Articulo No. 11 investigación con riesgo mayor que el mínimo, en el cual se informa el procedimiento, las características del proyecto a ejecutar y la confidencialidad de las mismas por consideraciones éticas.

Al momento del registro los consumidores debían informar el estado en que se encontraban para la realización de la prueba (sin consumo mayor a 24 horas o posterior al consumo), adicionalmente se preguntó a cada sujeto por antecedentes médicos, tanto consumidores como controles, y se descartaron aquellos que a lo largo de su vida, y por criterio médico reportaran padecer migraña, tumores, desmayos continuos, encefalopatías, traumatismos craneoencefálicos, enfermedades cerebrovasculares, tumores cerebrales, demencia, enfermedades degenerativas del SNC (Gil-Nagel et al. 2001), trastornos psiquiátricos o aquellos que tenían marcapasos, o platinas de metal en su cuerpo. 
Tabla 1. Características de la población

\begin{tabular}{|c|c|c|c|c|}
\hline & \multicolumn{2}{|c|}{$\begin{array}{l}\text { No Consumidores } \\
(\mathrm{N}=3)\end{array}$} & \multicolumn{2}{|c|}{$\begin{array}{c}\text { Consumidores } \\
\qquad(\mathrm{N}=16)\end{array}$} \\
\hline \multicolumn{5}{|c|}{ Género } \\
\hline Hombres N (\%) & \multicolumn{2}{|c|}{$1(33 \%)$} & \multicolumn{2}{|c|}{8 (50\%) } \\
\hline Mujeres N (\%) & \multicolumn{2}{|c|}{$2(67 \%)$} & \multicolumn{2}{|c|}{$8(50 \%)$} \\
\hline \multicolumn{5}{|c|}{ Edad (años) } \\
\hline Hombres $\mathrm{X} \pm \mathrm{SD}$ & \multicolumn{2}{|c|}{22} & \multicolumn{2}{|c|}{$26,12 \pm 1,80$} \\
\hline Mujeres $\mathrm{X} \pm \mathrm{SD}$ & \multicolumn{2}{|c|}{19} & \multicolumn{2}{|c|}{$23,10 \pm 2,77$} \\
\hline \multicolumn{5}{|c|}{ Estatura $(\mathrm{cm})$} \\
\hline Hombres $\mathrm{X} \pm \mathrm{SD}$ & \multicolumn{2}{|c|}{170} & \multicolumn{2}{|c|}{$175,3 \pm 7,31$} \\
\hline Mujeres $\mathrm{X} \pm \mathrm{SD}$ & \multicolumn{2}{|c|}{156} & \multicolumn{2}{|c|}{$160,6 \pm 6,38$} \\
\hline \multicolumn{5}{|c|}{ Peso (kg) } \\
\hline Hombres $X \pm S D$ & \multicolumn{2}{|c|}{60} & \multicolumn{2}{|c|}{$68 \pm 8,59$} \\
\hline Mujeres $\mathrm{X} \pm \mathrm{SD}$ & \multicolumn{2}{|c|}{57} & \multicolumn{2}{|c|}{$56 \pm 4,59$} \\
\hline \multicolumn{5}{|c|}{ Estrato } \\
\hline Estrato 1 & \multicolumn{2}{|c|}{0} & \multicolumn{2}{|c|}{1} \\
\hline Estrato 2 & \multicolumn{2}{|c|}{2} & \multicolumn{2}{|c|}{8} \\
\hline Estrato 3 & \multicolumn{2}{|c|}{1} & \multicolumn{2}{|c|}{5} \\
\hline Estrato 4 & & & & \\
\hline & acterística & consumo & & \\
\hline & Hombres & Mujeres & Hombres & Mujeres \\
\hline & Activic & & & \\
\hline Baja N (\%) & $1(100 \%)$ & $2(100 \%)$ & $4(50 \%)$ & $7(87,5 \%)$ \\
\hline Media N (\%) & - & - & $2(25 \%)$ & $1(12,5 \%)$ \\
\hline Alta N (\%) & - & - & $2(25 \%)$ & - \\
\hline & & & & \\
\hline No fumadores N (\%) & - & - & - & - \\
\hline Cannabis N (\%) & - & - & $4(50 \%)$ & $3(37,5 \%)$ \\
\hline Cannabis + Tabaco N (\%) & - & - & $4(50 \%)$ & $5(62,5 \%)$ \\
\hline & Años de & & & \\
\hline Bajo (1-5 Años) N (\%) & - & - & $2(25 \%)$ & $4(50 \%)$ \\
\hline Medio (6-10 Años) N (\%) & - & - & $2(25 \%)$ & $3(37,5 \%)$ \\
\hline Alto (11-15 Años) N (\%) & - & - & $4(50 \%)$ & $1(12,5 \%)$ \\
\hline & Frecuenci & umo & & \\
\hline Diario N (\%) & - & - & $5(62,5 \%)$ & $3(37,5 \%)$ \\
\hline Semanal N (\%) & - & - & $3(37,5 \%)$ & $4(50 \%)$ \\
\hline Mensual N (\%) & - & - & - & $1(12,5 \%)$ \\
\hline
\end{tabular}

La tabla presenta las características de la población seleccionada para el registro y análisis de EEG, indicando número de individuos por grupo poblacional, el porcentaje (\%) que representan. Se indica la media $(X)$ y desviación estándar (SD) de la edad, la estatura y el peso. Adicionalmente, se mencionan el número de personas y porcentaje, para las características (actividad física, consumo de tabaco) y hábitos de consumo en años (1-5; 6-10; 11-15) y en frecuencia (diario, semanal, mensual). 
A los usuarios de Cannabis se les solicitó que durante el período de participación en el estudio no emplearan más de tres psicoactivos (Cannabis, tabaco y alcohol). Conjuntamente, se sugirió para el registro con el efecto, que los hombres presentaran consumo de 1,0 a 1,5 gramos y mujeres de 0,5 a 1,0 gramo de Cannabis tipo corinto, la cual según Florián et al. (2009), es una de las variedades de Cannabis sativa que menor porcentaje tiene de THC con un 10,98\%, cuya biodisponibilidad (fracción de THC en el cigarrillo que pasa a sangre) está entre el $5 \mathrm{y}$ el 24 por ciento (entre $0,25 \mathrm{mg}$ y $30 \mathrm{mg}$ ), considerándose que para percibir un efecto significativo en consumidores ocasionales deben ser suministrados alrededor de 2 a $3 \mathrm{mg}$ (Sadock et al. 2007). Los registros se realizaron $10 \mathrm{~min}$ después de finalizado el consumo.

La tabla 1 presenta las características de la población seleccionada para el registro y análisis de EEG, indicando número de individuos por grupo poblacional, el porcentaje (\%) que representan. Se indica la media () y desviación estándar (SD) de la edad. Adicionalmente, se mencionan el número de personas y porcentaje, para las características y hábitos de consumo en años $(1-5 ; 6-10 ; 11-15)$ y en frecuencia (diario, semanal, mensual).

Se realizaron electroencefalogramas de monitorización pasiva, en la unidad de neurofisiología de la Clínica Palermo de Bogotá D.C., con un video electroencefalógrafo digital de 32 canales modelo EEG32 marca XLTEK (Versión 6.01) bajo el sistema internacional 10-20 modificado, empleando el software Natus Neuro Works, con el electrodo de referencia situado en la frente. Se registraron 15 minutos de EEG a cada sujeto, en posición decúbito supina, con ojos cerrados, en una habitación oscura, a una frecuencia de muestreo de $512 \mathrm{~Hz}$. Se descartaron 16 segundos de calibración y se exportaron a formato de texto (.txt) para su procesamiento.

El pre-procesamiento consistió en la implementación de filtros digitales, un filtro pasa bajo a $60 \mathrm{~Hz}$ para la eliminación de ruido de línea, un segundo filtro pasa bajo de $100 \mathrm{~Hz}$, para eliminación de frecuencias diferentes a la señal de EEG. Posteriormente se realizó la resta de los registros monopolares, para un total de 7 derivaciones (FP1-FP2, F7-F3, F3-FZ, FZ-F4, F4-F8, T3-C3, C4-T4), correspondientes a lóbulo Pre-Frontal, Frontal y Temporal. Se aplicó la transformada de Fourier, para la conversión de las señales al orden de frecuencia, y se implementaron filtros pasa banda para la extracción de la información de amplitud de las bandas de frecuencia, con un filtro butterworth de orden 4 inicial de 0,4 a $30 \mathrm{~Hz}$; para la extracción de los 4 ritmos de ondas, se empleó el mismo filtro, de igual orden, el ritmo beta se filtró de 13 a 30 $\mathrm{Hz}$, el ritmo alfa 8 a $13 \mathrm{~Hz}$, el ritmo theta de 4 a $8 \mathrm{~Hz}, \mathrm{y}$ el ritmo delta de 0,4 a $4 \mathrm{~Hz}$. Posteriormente, se extrajo la amplitud de la serie de Fourier total para la señal (de todos los ritmos de delta a beta), y se calculó la frecuencia media (FM) y el límite espectral al 95\% (SEF 95). Para la obtención de los resultados se contó con la asesoría del Doctor en Ingeniería Biomédica Daniel Alfonso Botero Rosas de la especialización en Bioingeniería de la Universidad Distrital Francisco José de Caldas y Oscar Leonardo Mosquera Dussan, candidato a Doctorado en Biociencias de la Universidad de la Sabana.

\section{RESULTADOS}

Los datos obtenidos se procesaron con el programa informático Statgraphics centurión XVI versión 16.1.18. Se aplicó la prueba de normalidad Kolmogórov-Smirnov encontrando que los datos eran normales con $\mathrm{p}>0.05$, permitiendo el análisis como estadística paramétrica. Se compararon las puntuaciones medias de dos grupos poblacionales: 1. estudiantes no consumidores (NC) contrastado con consumidores sin consumo (CSC), 2. estudiantes consumidores durante el efecto de Cannabis sativa (CCC) y sin consumo mayor a 24 horas (CSC); empleando la prueba t-Student para muestras independientes y pareadas respectivamente. Adicionalmente se realizó ANOVA de un factor para años y frecuencia de consumo; si el modelo ANOVA era significativo, se realizaban comparaciones múltiples post hoc con el test de Bonferroni. Se aceptó como grado de significancia estadística un valor de $p<0,05$. Cabe señalar que en los análisis no se realizó diferenciación por género, dado que no se realizarían pruebas de funciones cognitivas específicas. No obstante, se contó con la participación de igual número de hombres y mujeres.

Las áreas cerebrales aquí presentadas fueron seleccionadas teniendo en cuenta la bibliografía consultada, donde se menciona que el patrón de activación de los consumidores de Cannabis se asocia con la región pre-frontal, frontal y temporal, posiblemente relacionado con la alta densidad de receptores $\mathrm{CB} 1$ (receptor cannabinoide) en estas regiones (Fagundo et al. 2008).

Al contrastar el primer grupo poblacional mediante la prueba $\mathrm{t}$-student para muestras independientes, correspondiente a estudiantes $\mathrm{NC}$ con respecto a CSC, no se encontraron diferencias significativas para las variables 
de frecuencia delta, alfa y beta, en los lóbulos evaluados. La frecuencia theta presentó diferencias significativas para lóbulo temporal (tabla 2 y 3 ). En el análisis de ANOVA por factores (figura 1-10), no se encontraron diferencias significativas, para años y frecuencia de consumo entre grupos. En cuanto al segundo grupo poblacional de estudiantes CCC y CSC, al realizar la prueba t-student para muestras emparejadas, se encontraron diferencias significativas con un $p<0,05$ para todas las variables; en las frecuencias delta para lóbulo temporal; en frecuencias alfa para lóbulo frontal y temporal; en frecuencia beta para lóbulo frontal y temporal. No se encontraron diferencias significativas en frecuencias theta para ningún lóbulo (tabla 2 y 3). En el análisis de ANOVA por factores, no se encontraron diferencias significativas, para años y frecuencia de consumo entre grupos (figura 1-10)

Tabla 2. Resultados de las puntuaciones medias de NC, CSC y CCC

\begin{tabular}{|c|c|c|c|c|c|c|c|c|c|}
\hline \multirow{3}{*}{ Variables } & \multirow{2}{*}{\multicolumn{3}{|c|}{$\begin{array}{l}N C(3) \\
\bar{X}_{ \pm} S D\end{array}$}} & \multirow{2}{*}{\multicolumn{3}{|c|}{$\begin{array}{l}\operatorname{CSC}(16) \\
\bar{X}_{ \pm} S D\end{array}$}} & \multirow{2}{*}{\multicolumn{3}{|c|}{$\begin{array}{l}C C C(16) \\
\bar{X}_{ \pm S D}\end{array}$}} \\
\hline & & & & & & & & & \\
\hline & PF & $\mathrm{F}$ & $\mathrm{T}$ & $\mathrm{PF}$ & $\mathrm{F}$ & $\mathrm{T}$ & $\mathrm{PF}$ & $\mathrm{F}$ & $\mathrm{T}$ \\
\hline F. Delta (\%) & $\begin{array}{c}0,861 \pm \\
0,053\end{array}$ & $\begin{array}{c}0,733 \pm \\
0,196\end{array}$ & $\begin{array}{c}0,521 \pm \\
0,009\end{array}$ & $\begin{array}{c}0,898 \pm \\
0,072\end{array}$ & $\begin{array}{c}0,826 \pm \\
0,082\end{array}$ & $\begin{array}{c}0,648 \pm \\
0,153\end{array}$ & $\begin{array}{c}0,891 \pm \\
0,086\end{array}$ & $\begin{array}{c}0,784 \pm \\
0,096\end{array}$ & $\begin{array}{c}0,575 \pm \\
0,178\end{array}$ \\
\hline F. Theta (\%) & $\begin{array}{c}0,068 \pm \\
0,029\end{array}$ & $\begin{array}{c}0,079 \pm \\
0,035\end{array}$ & $\begin{array}{c}0,178 \pm \\
0,073\end{array}$ & $\begin{array}{c}0,050 \pm \\
0,048\end{array}$ & $\begin{array}{c}0,072 \pm \\
0,033\end{array}$ & $\begin{array}{c}0,114 \pm \\
0,038\end{array}$ & $\begin{array}{c}0,037 \pm \\
0,021\end{array}$ & $\begin{array}{c}0,070 \pm \\
0,027\end{array}$ & $\begin{array}{c}0,096 \pm \\
0,042\end{array}$ \\
\hline F. Alfa (\%) & $\begin{array}{c}0,024 \pm \\
0,006\end{array}$ & $\begin{array}{c}0,052 \pm \\
0,010\end{array}$ & $\begin{array}{c}0,159 \pm \\
0,092\end{array}$ & $\begin{array}{c}0,019 \pm \\
0,014\end{array}$ & $\begin{array}{c}0,045 \pm \\
0,027\end{array}$ & $\begin{array}{c}0,109 \pm \\
0,067\end{array}$ & $\begin{array}{c}0,023 \pm \\
0,019\end{array}$ & $\begin{array}{c}0,068 \pm \\
0,048\end{array}$ & $\begin{array}{c}0,167 \pm \\
0,130\end{array}$ \\
\hline F. Beta (\%) & $\begin{array}{c}0,047 \pm \\
0,040\end{array}$ & $\begin{array}{c}0,114 \pm \\
0,130\end{array}$ & $\begin{array}{c}0,141 \pm \\
0,037\end{array}$ & $\begin{array}{c}0,033 \pm \\
0,028\end{array}$ & $\begin{array}{c}0,057 \pm \\
0,040\end{array}$ & $\begin{array}{c}0,129 \pm \\
0,116\end{array}$ & $\begin{array}{c}0,050 \pm \\
0,055\end{array}$ & $\begin{array}{c}0,078 \pm \\
0,052\end{array}$ & $\begin{array}{c}0,162 \pm \\
0,129\end{array}$ \\
\hline
\end{tabular}

Se presenta la media ( $\bar{X}$ y desviación estándar (SD) de cada una de las variables evaluadas (frecuencias delta, theta, alfa y beta ) de las poblaciones estudiadas: No consumidores (NC), consumidores sin consumo (CSC) y con consumo (CCC) para cada uno de los lóbulos evaluados, Pre Frontal (PF), Frontal (F) y Temporal (T).

Tabla 3. Resultados significancia prueba $t$-student

\begin{tabular}{ccccccc}
\hline \multirow{2}{*}{ Variables } & \multicolumn{3}{c}{ NC } & Vs CSC & \multicolumn{3}{c}{ CSC Vs CCC } \\
& PF & F & T & PF & F & T \\
\hline F. Delta & 0,412 & 0,501 & 0,180 & 0,754 & 0,075 & $0,040^{*}$ \\
F. Theta & 0,099 & 0,751 & $0,033^{*}$ & 0,259 & 0,687 & 0,138 \\
F. Alfa & 0,542 & 0,670 & 0,267 & 0,323 & $0,033^{*}$ & $0,024^{*}$ \\
F. Beta & 0,450 & 0,527 & 0,860 & 0,151 & $0,030^{*}$ & $0,025^{*}$
\end{tabular}

Se presentan los datos de significancia de las comparaciones realizadas en la prueba $t$-student, entre no consumidores (NC) y consumidores sin consumo (CSC), y entre CSC y consumidores con consumo (CCC) para cada uno de los lóbulos evaluados, Pre Frontal (PF), Frontal (F) y Temporal (T). Los valores que presentan significancia estadística con un $\mathrm{p}<0,05$ se señalan con un *. 


\section{Análisis por Factores}

A continuación, se presentan gráficamente los resultados anteriormente mencionados del análisis por los factores: años y frecuencia de consumo.

\section{Factor Años de Consumo}

\section{Bandas de frecuencia Delta}

Bandas de frecuencia Alfa

Factor Frecuencia De Consumo

Bandas de Frecuencia Alfa

Bandas de Frecuencia Beta

\section{DISCUSIÓN}

\section{Frecuencia Delta}

En el presente estudio se observaron diferencias significativas en la comparación entre no consumidores con respecto a consumidores sin el efecto de Cannabis (tabla 2 y 3 ) en las frecuencias delta para lóbulo temporal, siendo mayor en ausencia de consumo, a su vez, en el análisis por factores a pesar de no presentar significancia estadística, durante el consumo se pudo observar una relación proporcional con respecto a los años de consumo (figura 1 - 3), incrementándose la frecuencia delta en los lóbulos prefrontal, frontal y temporal en los consumidores de mayor tiempo de exposición a la sustancia. Lo anterior se relaciona con los resultados encontrados por Herning et al. (2008), que indicaron un aumento en la actividad delta en el EEG de consumidores de Cannabis acompañado por déficits más grandes en la perfusión cerebral. Así mismo en una investigación realizada por Herning et al. (2008) se reportó que la frecuencia delta fue elevada en la mayoría de áreas registradas del cerebro de los abusadores de Cannabis con respecto a no consumidores, lo cual se complementa con lo expresado por Ehlers et al. (2010), quienes indican un aumento de la actividad delta frontal en los individuos con dependencia a Cannabis y abstinencia mayor a 30 días asociado a un leve deterioro cognitivo y de la memoria.

Por otro lado, en un estudio realizado por Herning et al. (2008), en 75 usuarios de Cannabis con abstinencia de 30 días y 33 controles, durante 72 horas de registro electroencefalográfico, se presentaron aumentos en la frecuencia delta, indicando que los usuarios de Cannabis podrían estar más somnolientos que los sujetos control ya que estos reportaban problemas para dormir durante la abstinencia temprana. Los cambios observados en la frecuencia delta indican que los consumidores de Cannabis presentan un incremento de ésta en ausencia de consumo, lo cual se relaciona con lo planteado por Knyazev (2012), quien expresa que las ondas delta aumentan en estados de ansiedad producto de la abstinencia y disminuye tras la recompensa generada con el consumo, sugiriendo además que la liberación de dopamina durante el efecto de la sustancia conduce a la disminución de las ondas delta.

\section{Frecuencia Theta}

En cuanto a la comparación de estudiantes consumidores durante y en ausencia de consumo (tabla 2 y 3 ) en la frecuencia Theta para lóbulo temporal, siendo mayor en consumidores sin consumo, lo cual concuerda con lo reportado por Herning et al. (2008), quienes indican que en los consumidores de Cannabis se aumenta la actividad de la frecuencia theta tras el consumo, complementado con la investigación desarrollada por Struve et al. (1996), en una población de larga y moderada duración de consumo contrastado con población no consumidora, empleando un

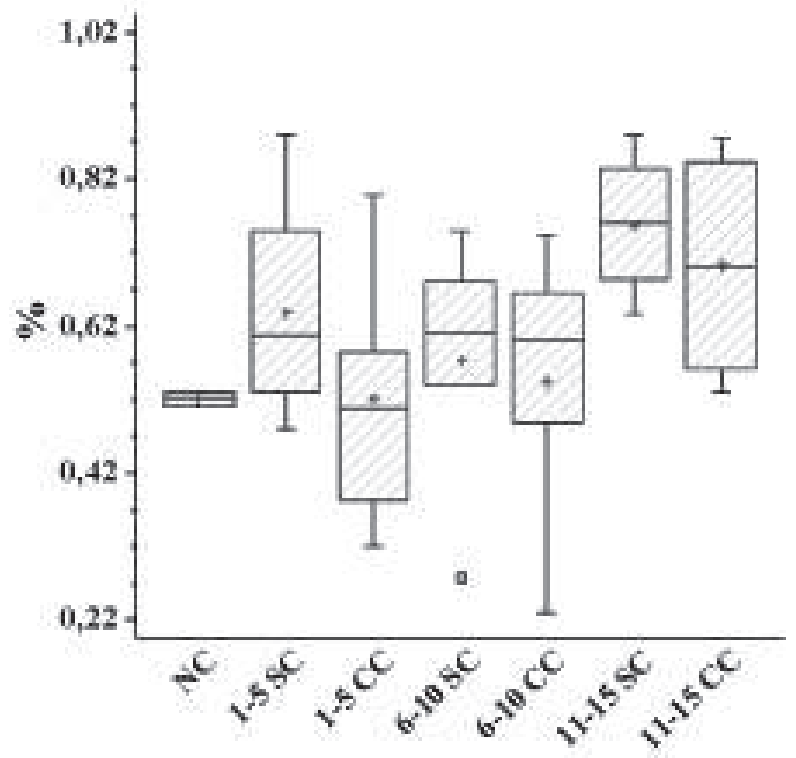

Figura 1. Comparación de la frecuencia delta en lóbulo temporal para el factor años de consumo. El gráfico ilustra la mediana, desviación estándar, valores máximos y mínimos, entre cada uno de los grupos: No consumidores (NC), consumidores de 1-5 años, 6-10 años y 11-15 años de consumo, sin consumo (SC) y durante el consumo (CC). 


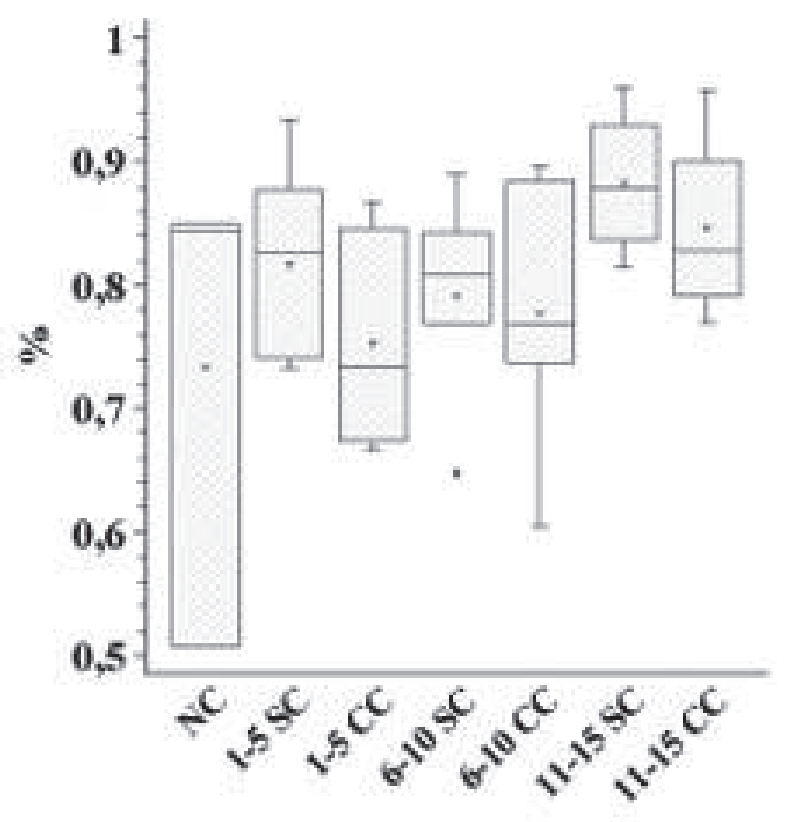

Figura 2. Comparación de la frecuencia delta en lóbulo frontal para el factor años de consumo. El gráfico ilustra la mediana, desviación estándar, valores máximos y mínimos, entre cada uno de los grupos: No consumidores (NC), consumidores de 1-5 años, 6-10 años y 11-15 años de consumo, sin consumo (SC) y durante el consumo (CC).

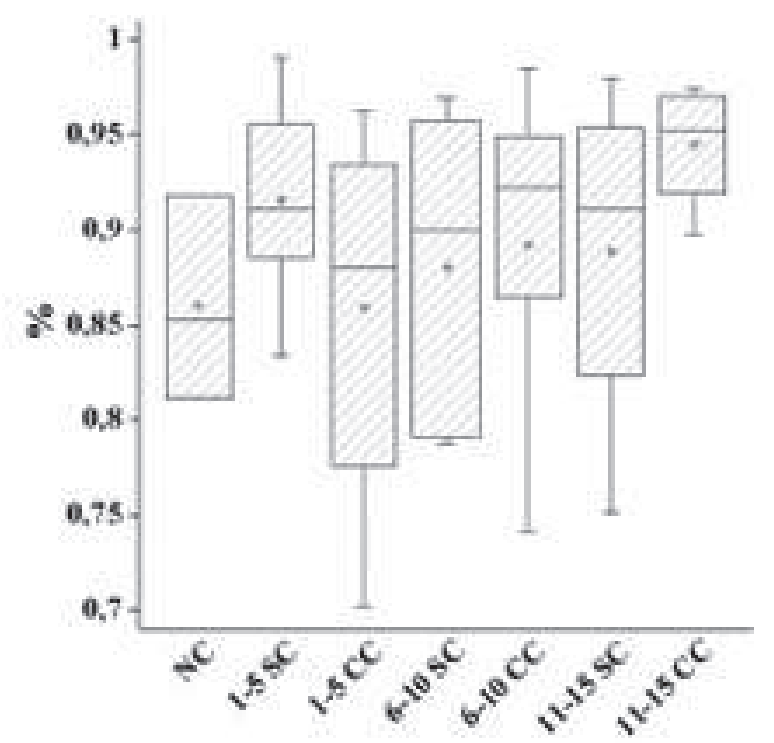

Figura 3. Comparación de la frecuencia delta en lóbulo pre-frontal para el factor años de consumo. El gráfico ilustra la mediana, desviación estándar, valores máximos y mínimos, entre cada uno de los grupos: No consumidores (NC), consumidores de 1-5 años, 6-10 años y 11-15 años de consumo, sin consumo (SC) y durante el consumo (CC).
EEG cuantitativo topográfico que evidenció una elevada actividad theta para todos los lóbulos de consumidores de larga duración con respecto a moderados y controles. En contradicción, Ilan et al. (2004), expresan que fumar Cannabis disminuye la frecuencia theta, relacionado con un aumento de la activación autonómica, y a su vez Hart et al. (2010), añaden que existe una reducción de los efectos de la frecuencia theta 15 min después del consumo de Cannabis. Los incrementos de la actividad theta en consumidores (tanto en ausencia como durante los efectos del consumo) con respecto a los no consumidores pueden reflejar deficiencia en el procesamiento de información (Quesada et al. 2007) y pueden constituir un índice electrofisiológico de desequilibrio entre los mecanismos de excitación e inhibición de la corteza (Quesada et al. 2007) que se manifiestan en estados de somnolencia, adormecimiento, relajación profunda y percepción reducida (Arbaiza del Rio 2014).

\section{Frecuencia Alfa}

Con respecto a la frecuencia alfa (tabla 2 y3), evaluada en el presente estudio se observaron diferencias significativas con un incremento durante el consumo de Cannabis en lóbulo frontal y temporal; es de resaltar que, aunque el análisis por factores no presentó significancia estadística, se evidenció una disminución en las frecuencias alfa de los consumidores de 11 a 15 años para los 3 lóbulos evaluados (figura 4 y 5). La frecuencia de consumo presentó una relación lineal para las frecuencias alfa, siendo menor en los lóbulos prefrontal, frontal y temporal en aquellas personas que consumían diariamente (figura 6 - 8).

Lo cual concuerda con lo planteado por Cohen (1986), Jones (1980) y Klonoff (1973) citado por Nelson (1993), quienes indican aumento de la actividad alfa de consumidores de Cannabis durante el efecto, asociado con estados de somnolencia, relajación, depresión subconsciente o ansiedad (Struve et al. 1998, Fernandez-Guardiola et al. 1976). Así mismo, Struve et al. (1989, 1994), observaron un aumento significativo en el poder absoluto, el poder relativo y la coherencia interhemisférica de actividad alfa sobre la corteza frontal central bilateral en consumidores diarios de Cannabis, en contraste con los no consumidores, y a su vez, aumentos significativos transitorios relacionados con la dosis en el poder alfa y la desaceleración de esta frecuencia después de la exposición aguda de Cannabis, la cual decae a los niveles basales en 4 horas (Struve et al. 1996). Esto indica que, durante el efecto de Cannabis, las ondas alfa se incrementan, siendo característico de estados de relajación, poca atención y concentración, así mismo 


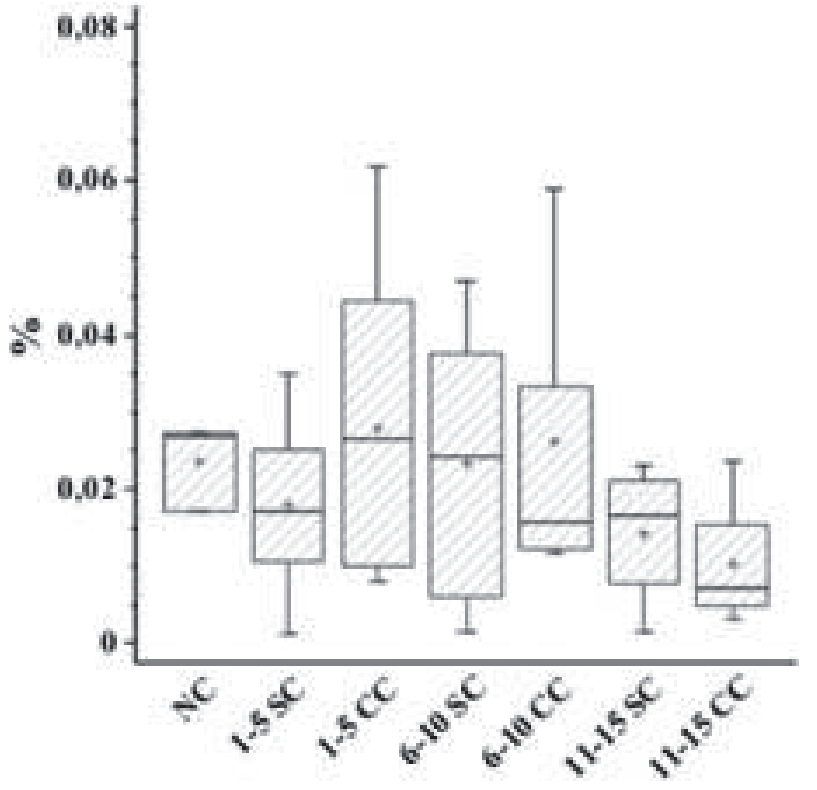

Figura 4. Comparación de la frecuencia alfa en lóbulo prefrontal para el factor años de consumo. El gráfico ilustra la mediana, desviación estándar, valores máximos y mínimos, entre cada uno de los grupos: No consumidores (NC), consumidores de 1-5 años, 6-10 años y 11-15 años de consumo, sin consumo (SC) y durante el consumo (CC).

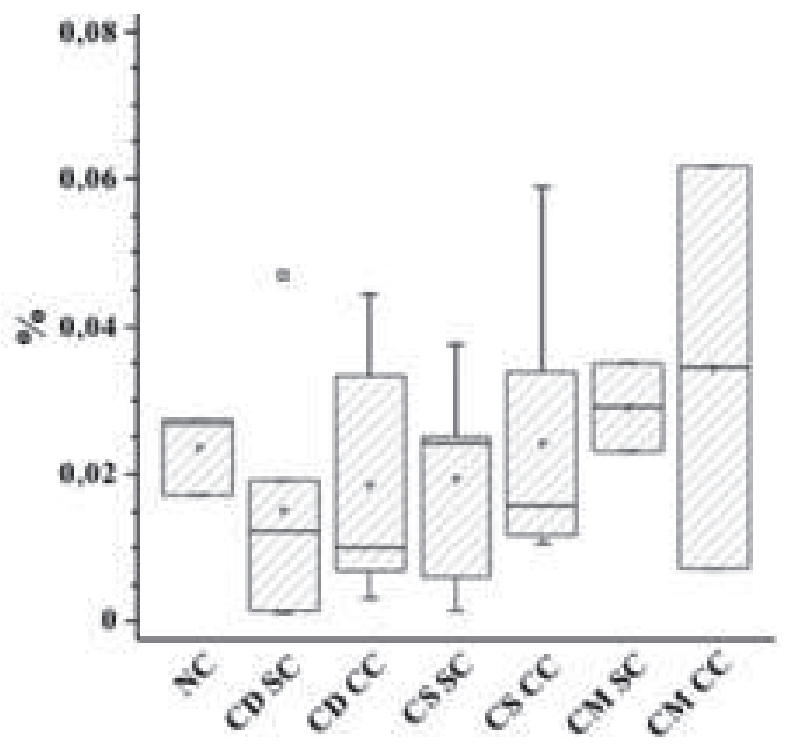

Figura 6. Comparación de la frecuencia alfa en lóbulo prefrontal para el factor frecuencia de consumo. El gráfico ilustra la mediana, desviación estándar, valores máximos y mínimos, entre cada uno de los grupos: No consumidor (NC) Consumidor Diario (CD), Semanal (CS), QuincenalMensual (CM), sin consumo (SC) y Con consumo (CC).

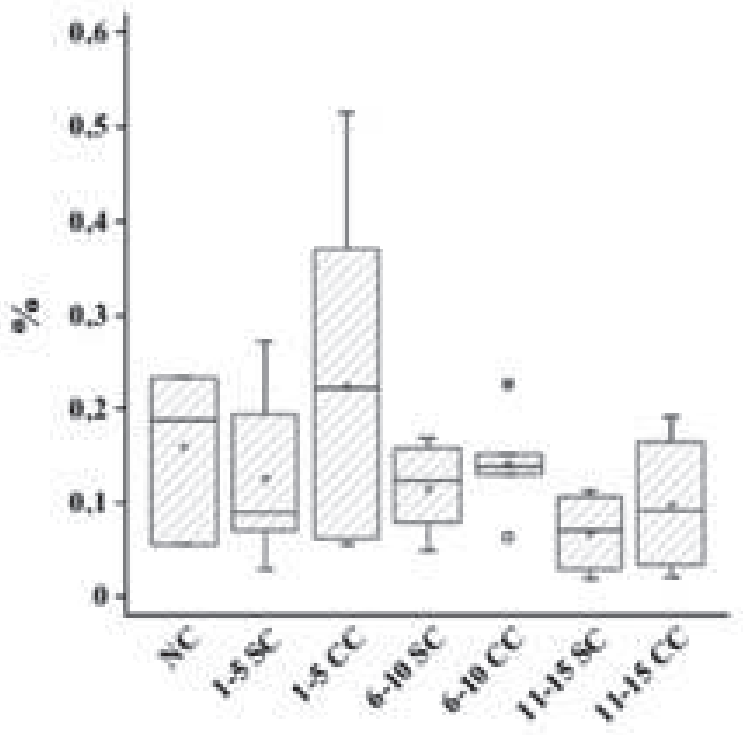

Figura 5. Comparación de la frecuencia alfa en lóbulo temporal para el factor años de consumo. El gráfico ilustra la mediana, desviación estándar, valores máximos y mínimos, entre cada uno de los grupos: No consumidores (NC), consumidores de 1-5 años, 6-10 años y 11-15 años de consumo, sin consumo (SC) y durante el consumo (CC).

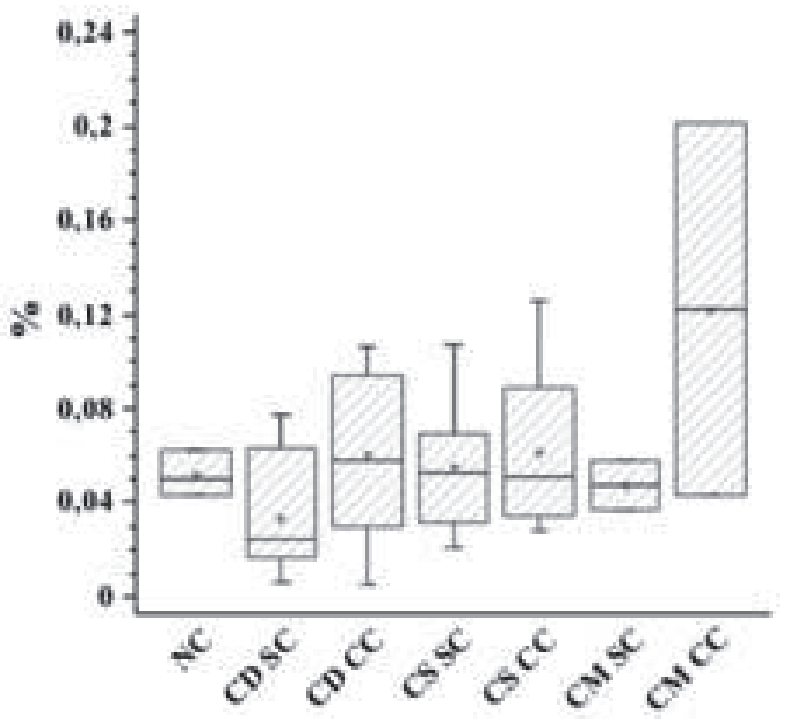

Figura 7. Comparación de la frecuencia alfa en lóbulo frontal para el factor frecuencia de consumo. El gráfico ilustra la mediana, desviación estándar, valores máximos y mínimos, entre cada uno de los grupos: No consumidor (NC) Consumidor Diario (CD), Semanal (CS), QuincenalMensual (CM), sin consumo (SC) y Con consumo (CC). 


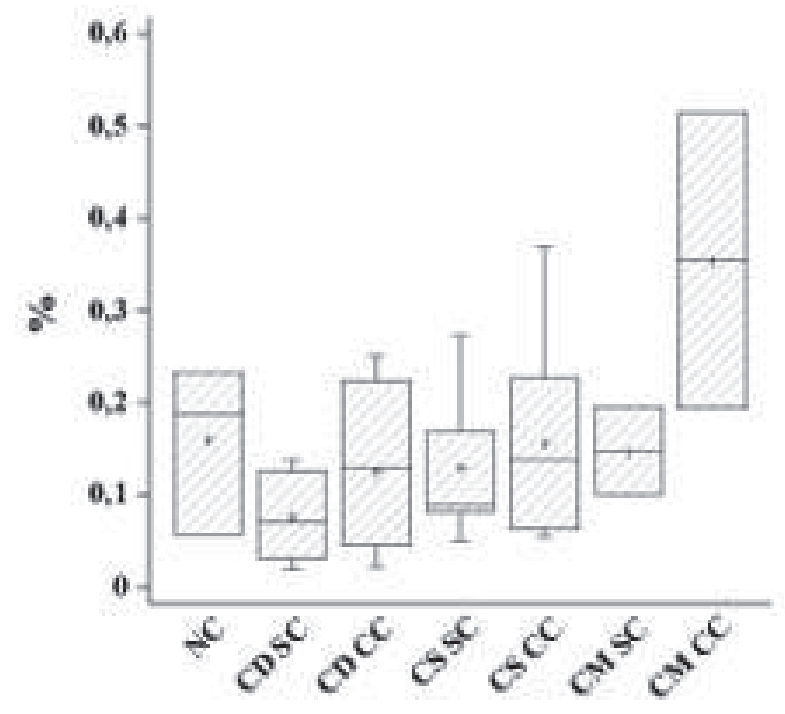

Figura 8. Comparación de la frecuencia alfa en lóbulo temporal para el factor frecuencia de consumo. El gráfico ilustra la mediana, desviación estándar, valores máximos y mínimos, entre cada uno de los grupos: No consumidor (NC) Consumidor Diario (CD), Semanal (CS), QuincenalMensual (CM), sin consumo (SC) y Con consumo (CC).

Bazanova y Vernon (2014), informan que el aumento de la potencia alfa se relaciona con la disminución de la sangre oxigenada, indicando inactividad cortical.

Por otro lado, Ceballos et al. (2009) y Herning et al. (2008), informaron una asociación entre la duración del tiempo de consumo y la reducción de la frecuencia alfa, en los abusadores de Cannabis que la usaron durante ocho años o más. Lo cual se complementa con lo planteado por Struve et al. (1993), quienes identificaron una disminución significativa de la frecuencia alfa relacionada con la exposición crónica a Cannabis. Lo anterior podría sugerir que con el uso prolongado de Cannabis los consumidores generan una tolerancia a la sustancia, producido por cambios en su absorción, distribución, biotransformación y excreción; adicionalmente, se ha propuesto una activación del complejo del citocromo P450 que facilitaría la degradación de los cannabinoides. No obstante, los principales estudios han demostrado que la tolerancia tiene preferentemente una base farmacodinámica, consecuencia de la disminución de la densidad, afinidad y/o eficacia de los receptores CB1, presentes en las regiones cerebrales implicadas en los efectos farmacológicos de los cannabinoides. Algunos estudios a nivel molecular sugieren que el inicio de la tolerancia farmacodinámica ocurre por desensibilización de los receptores $\mathrm{CB} 1$ como consecuencia de su fosforilación y posterior secuestro por arrestinas en el interior celular, si esta situación persiste, el fenómeno deriva en degradación de los receptores internalizados, siendo necesaria la síntesis de nuevos receptores, aunque si la situación se revierte a corto plazo, los receptores internalizados podrían volver a la membrana (Sagredo 2011), situación que sería más evidente en los consumidores de mayor tiempo y exposición a la sustancia.

La afectación de la actividad en las áreas prefrontales, explicaría las alteraciones en atención, concentración, capacidad de juicio, así como apatía o pasividad que acompañan los estadios de abstinencia al Cannabis (Fagundo et al. 2008). A su vez, como el lóbulo frontal es responsable directa o indirectamente las funciones ejecutivas, puesto que supervisan y coordinan las actividades relacionadas con inteligencia, atención, memoria, lenguaje, flexibilidad mental, control motor y regulación de la conducta emocional (Fagundo et al. 2008), que se constituyen como disfunciones en los consumidores de Cannabis.

\section{Frecuencia Beta}

La frecuencia beta (tabla 2 y 3 ) analizada en la presente investigación, presentó diferencias estadísticamente significativas, con un incremento durante el consumo en lóbulo frontal y temporal. Aunque el análisis por factores no tuvo significancia estadística, para los años de consumo se denotó una disminución de la frecuencia beta para los lóbulos prefrontal y temporal en los consumidores de 11 a 15 años. Con respecto a la frecuencia de exposición a la sustancia, se evidenció en lóbulo prefrontal un aumento en la frecuencia beta en los consumidores diarios, contrario a lo registrado en lóbulo frontal donde se incrementó en los consumidores crónicos (figura 9 y 10). Lo cual concuerda con Ilan et al. (2004), Herning et al. (2008) y Ceballos et al. (2009), quienes reportan que los consumidores de mayor tiempo de consumo presentaron una disminución de la frecuencia beta, con respecto a los consumidores de menor tiempo de consumo y los controles, complementado por Hart et al. (2010), quienes expresan que el aumento de la frecuencia beta se reduce 15 minutos después de fumar Cannabis, incrementándose cuando se disipan los efectos de la sustancia. Esta frecuencia es habitual del estado de vigilia y se asocia con el pensamiento y la atención activa, o en la resolución de problemas concretos, por lo cual, el aumento de ésta durante el consumo podría sugerir actividad cerebral. Sin embargo, durante el consumo prolongado la presencia de dicha onda decrece, indicando 
menor actividad cerebral, lo cual se corresponde con el aumento en la frecuencia delta y theta en ausencia de consumo, anteriormente mencionados.

En conclusión, se puede mencionar que entre los efectos de Cannabis en la actividad eléctrica cerebral durante el consumo están: la presencia de una mayor actividad alfa, indicando estados de relajación y poca concentración; un incremento en la actividad beta, vinculada a la captación de diversos estímulos; disminución de la actividad delta, asociada con estados de ansiedad. A consecuencia de la abstinencia en ausencia de consumo, se resalta el aumento de las ondas delta con respecto a los no consumidores, la cual disminuye tras la recompensa generada con el consumo. Adicionalmente, se evidenció que el consumo prolongado y frecuente de esta sustancia genera una tolerancia, al no variar la actividad cerebral durante y en ausencia del efecto de Cannabis sativa; del mismo modo, la presencia en mayor proporción de ondas theta en ausencia de consumo frente a no consumidores, propone que, tras el uso prolongado de la sustancia, los consumidores tienden a manifestar estados de somnolencia y adormecimiento.

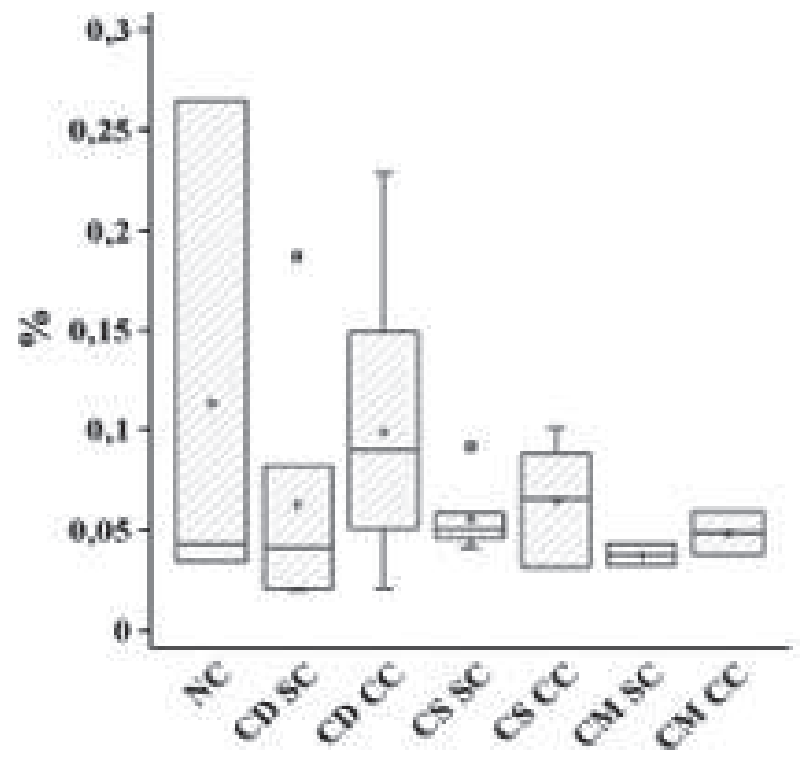

Figura 9. Comparación de la frecuencia beta en lóbulo frontal para el factor frecuencia de consumo. El gráfico ilustra la mediana, desviación estándar, valores máximos y mínimos, entre cada uno de los grupos: No consumidor (NC) Consumidor Diario (CD), Semanal (CS), QuincenalMensual (CM), sin consumo (SC) y Con consumo (CC).

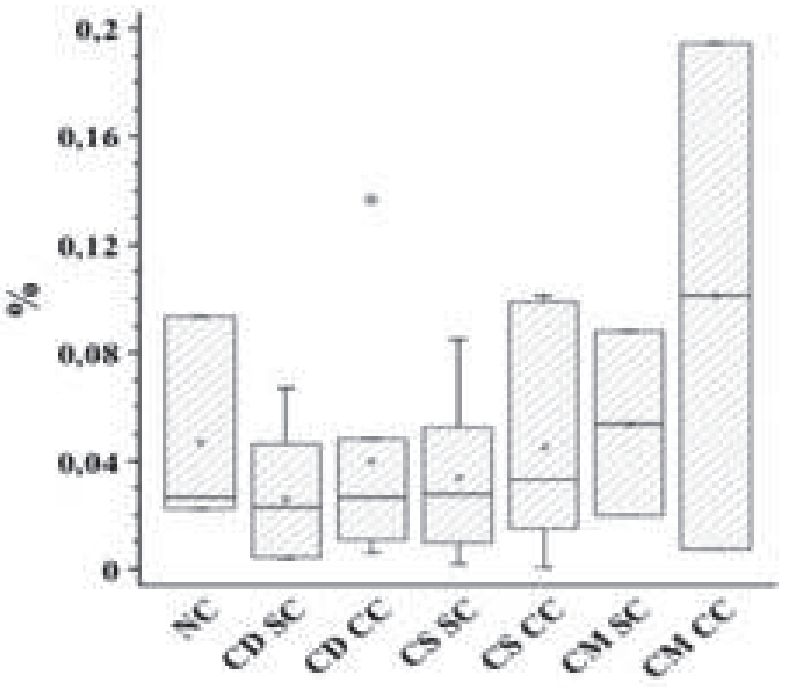

Figura 10. Comparación de la frecuencia beta en lóbulo pre-frontal para el factor frecuencia de consumo. El gráfico ilustra la mediana, desviación estándar, valores máximos y mínimos, entre cada uno de los grupos: No consumidor (NC) Consumidor Diario (CD), Semanal (CS), Quincenal- Mensual (CM), sin consumo (SC) y Con consumo (CC).

\section{AGRADECIMIENTOS}

El presente estudio se realizó en el marco del macro proyecto "efectos del consumo de Cannabis sativa en los sistemas nervioso, circulatorio, respiratorio y endocrino e influencia en la capacidad cognitiva de consumidores universitarios habituales", desarrollado por el grupo de investigación en Neurociencias GINUD, gracias a la financiación del Centro de Investigaciones y Desarrollo Científico de la Universidad Distrital Francisco José de Caldas (CIDC) y al aval del Comité de Bioética para la Investigación de la Universidad Distrital. A la Universidad Distrital en especial al Proyecto Curricular de Licenciatura en Biología. A la unidad de neurofisiología de la Clínica Palermo por apoyar la realización de los registros electroencefalográficos. Al Doctor Daniel Alfonso Botero Rosas, Ph.D. de la Universidade Federal Do Rio De Janeiro, al Candidato a Doctor de la Universidad de La Sabana, Oscar Leonardo Mosquera Dussán y al Especialista en Bioingeniería de la Universidad Distrital Francisco José de Caldas, Cristian Javier Murcia Garzón, por sus aportes en las metodologías y en los procedimientos de obtención de resultados. A los evaluadores que contribuyeron a la edición final de este artículo. 


\section{REFERENCIAS}

Arbaiza del Rio M. 2014. Alteraciones cognitivas, conectividad funcional y personalidad en el drogodependiente [Tesis doctoral]. [Madrid (España)]: Facultad de Psicología, Departamento de psicología básica II, Universidad Complutense de Madrid. p. 111.

Bazanova O y Vernon D. 2014. Interpreting EEG Alpha Activity. Neuroscience and Biobehavioral Reviews, 44: 94-110.

Ceballos N, Bauer L, Houston R. 2009. Recent EEG and ERP Findings in Substance Abusers. Clinical EEG and Neuroscience, 40 (2): 122-128.

Constant I, Sabourdin N. 2012. The EEG signal: a window on the cortical brain activity. Pediatric Anesthesia, 22 (6): 539-532.

Ehlers C, Phillips E, Gizer I, Gilder D, Wilhelmsen K. 2010. EEG spectral phenotypes: Heritability and association with marijuana and alcohol dependence in an American Indian community study. Drug and Alcohol Dependence, 106 (2-3): 101-110.

Fagundo A, Martín-Santos R, Abanades S, Farré M, Verdejo-García A. 2008. Neuroimagen y adicción II: Correlatos neuroanatómicos y funcionales de la administración aguda, el craving y el consumo crónico de opiáceos, alcohol y Cannabis. Revista Española de Drogodependencia, 33 (2): 125-149.

Franco M. 2007. Manual de Electrocardiografía y Electroencefalografía. México: Manual Moderno. p. 281.

Gil-Nagel A, Parra J, Iriarte J, Kenner A. 2001. Manual de electroencefalografía. Madrid (España): McGraw-Hill/ Interamericana de España. p. 499.

Fernandez-Guardiola A, Salgado A, Contreras CM, Condes M, GonzalesEstrado MT, Solis H, Calvo JM, Ayala F. 1976. Multiunit Activity and Polygraphic Recordings of the Pharmacological Effects of delta 9-Tetrahydrocannabinol. En: The Pharmacology of Marihuana. Braude MC and Szara S editors. New York: Raven Press. p. 335-344.

Hart C, Ilan A, Gevins A, Gunderson E, Role K, Colley J, Foltin R. 2010. Neurophysiological and cognitive effects of smoked marijuana in frequent users. Pharmacology Biochemistry and Behavior, 96 (3): 333-341.

Herning R, Better W, Cadet J. 2008. EEG of chronic marijuana users during abstinence: Relationship to years of marijuana use, cerebral blood flow and thyroid function. Clinical Neurophysiology, 119 (2): 321-331.

Ilan A, Smith M, Gevins A. 2004. Effects of marijuana on neurophysiological signals of working and episodic memory. Psychopharmacology, 176 (2): 214-222.
Knyazev G. 2012. EEG delta oscillations as a correlate of basic homeostatic and motivational processes. Neuroscience and Biobehavioral Reviews, 36 (1), 677-695.

Nelson P. 1993. A critical review of the research literature concerning some biological and psychological effects of Cannabis. Cannabis and the law in Queensland:Adiscussion paper. [Internet], 113-152. Fecha de acceso: 01 de enero de 2015. Disponible en: $<$ http://www.ukcia.org/research/ ReviewOfResearchLiteratureConcerningEffectsOfCannabis.php>.

Quesada M, Díaz G, Herrera A, Tamayo M, Rubio R. 2007. Características del electroencefalograma cuantitativo y trastornos cognitivos en pacientes alcohólicos. Revista de Neurología, 44: 81-88.

Sadock B, Kaplan H, Sadock V. 2007. Substance-Related Disorders: Introduction and Overview. En: Synopsis of Psychiatry: Behavioral Sciences/Clinical Psychiatry. 10a Edición. Philadelphia: Wolters Kluwer; Lippincott Williams y Wilkins. p. 381-464.

Sagredo O. 2011. Efectos farmacológicos y fisiológicos del consumo de Cannabis. Trastornos Adictivos, 13 (3): 94-96.

Sanei S, Chambers J. 2007. EEG signal proccesing. Inglaterra: Jhon Wiley and Sons editores. p. 312.

Struve F, Straumanis J, Patrick G, Price L. 1989. Topographic mapping of quantitative EEG variables in heavy marihuana users: empirical findings with psychiatric patients. Clinical Electroencephalogram, 20 (1): 6-23.

Struve F, Straumanis J, Patrick G. 1993. Altered quantitative EEG topography as sequelae of chronic THC exposure: a replication using screened normals. En: Harris L (ed). Problems of Drug Dependence 1992, National Institute on Drug Abuse. Research Monograph No. 132. Washington DC: US Government Printing Office, 132.

Struve F, Straumanis J, Patrick G. 1994. Persistent topographic quantitative EEG sequelae of chronic marihuana use: a replication study and initial discriminant function analysis. Clinical Electroencephalogram, 25 (2): 63-75.

Struve F, Manno B, Kemp P, Patrick G. 1996. Change in quantitative EEG "alpha hyperfrontality" during and following inhalation of THC. En: Harris L. Problems of drug dependence. Proceedings of the 57th annual scientific meeting, college on problems of drug dependence. NIDA Research Monograph No. 162 NIH Publication No. 96-4116, p. 292.

Struve F, Patrick G, Straumanis J, Fitz-Gerald M, Manno J. 1998. Possible EEG sequelae of very long duration marihuana use: pilot findings from topographic quantitative EEG analyses of subjects with 15 to 24 years of cumulative daily exposure to THC. Clinical Electroencephalography, 29 (1): 31-36. 